\title{
Dynamical core deformation effects on single-nucleon knockout reactions at fragmentation beam energies
}

\author{
P. Batham, I. J. Thompson, and J. A. Tostevin* \\ Department of Physics, School of Electronics and Physical Sciences, University of Surrey, Guildford, Surrey GU2 7XH, United Kingdom
}

(Received 11 January 2005; published 28 June 2005)

\begin{abstract}
The effects of core deformation and of its dynamical reorientation and rotational excitation on the inclusive single-neutron knockout reaction cross sections on light spherical target nuclei are examined. The projectile nuclei are modeled within the framework of a weak-coupling, quadrupole-deformed core-plus-neutron two-body model. We formulate the inclusion of this non-spectator-core degree of freedom within the nonperturbative eikonal model and calculate the elastic and inelastic breakup (or stripping) neutron-removal cross sections. We apply the methods to model the single-neutron removal reactions induced by ${ }^{11} \mathrm{Be}$ and ${ }^{17} \mathrm{C}$ secondary fragmentation beams incident on a ${ }^{9} \mathrm{Be}$ target. Our calculations indicate that dynamical deformation effects on the elastic breakup component of the knockout cross section can be significant. This is the largest effect. The more geometrical stripping cross section is found to be hardly changed by the inclusion of the deformed core degree of freedom.
\end{abstract}

DOI: 10.1103/PhysRevC.71.064608 PACS number(s): 21.10.Jx, 24.10.-i, 25.60.Gc, 27.20.+n

\section{INTRODUCTION}

Single-nucleon knockout reactions, initiated by secondary beams of short-lived exotic nuclei at fragmentation beam energies, have significant cross sections and have been shown to be a powerful tool for studying the structure of light- and medium-mass nuclei [1,2]. In almost all cases the reaction populates a number of hole states in the reaction residue leading to sizable cross sections to several discrete, bound residue excited states. The knockout method is therefore at its most powerful when measurements are made of coincidences between the reaction residues and their decay $\gamma$ rays to obtain partial cross sections to one or more such final states.

To date, essentially all calculations have made the spectatorcore approximation [3,4] in which it is assumed that the internal degrees of freedom of these reaction residues are inert and are not dynamically excited or deexcited during the reaction. This allows a direct structural connection to be made between the measured partial cross sections and the wave function overlaps between the projectile initial state and the measured residue final states. The importance of dynamical core state excitation effects, associated with single-particle excitations or the deformation degree of freedom, remain to be fully clarified. Calculations of single-particle-like inelastic terms, in Ref. [4], suggested that single-nucleon excitation cross sections were small. This view was supported by an estimate of the effects on the stripping cross section [5]. Conversely, an earlier estimate was made of collective, core rotational excitation in the case of neutron knockout from ${ }^{11} \mathrm{Be}[6]$, where a contribution from $0^{+} \rightarrow 2^{+}$state inelastic excitation of ${ }^{10} \mathrm{Be}$ was significant and was needed to explain an enhanced ${ }^{10} \mathrm{Be}\left(2^{+}\right)$final state partial cross section. There the changes were anticipated as arising principally from an enhanced elastic breakup component.

In this article we perform a first investigation of the effects of the dynamical excitation and also reorientation

*Electronic address: j.tostevin@surrey.ac.uk of an assumed quadrupole-deformed projectile core on both the elastic breakup and the stripping components of the inclusive single-nucleon knockout cross sections. A closely related analysis, of the effects of deformation of the core and of the removed nucleon's single-particle state, was reported previously by Sakharuk and Zelevinsky [7]. Sakharuk and Zelevinsky calculated the cross section from the stripping mechanism only, using the strong-coupling (Nilsson basis) approach. They found the associated deformation effects on the nucleon stripping cross section to be relatively small.

We consider here nucleon knockout from weakly bound projectiles where the breakup mechanism is expected to play an important role. Such systems have been reasonably well modeled (e.g., Ref. [8] and references therein) as bound coupled-channels eigenstates of the deformed core with the loosely bound neutron. Unlike the deformed-basis analysis of Ref. [7], our approach uses a spherical single-particle basis. In Sec. II we discuss the generalization of the reaction formalism required to incorporate the core deformation degree of freedom and in Sec. III the treatment of the core- and nucleon-target $S$ matrices. Section IV then presents the model results for applications to neutron knockout reactions from ${ }^{11} \mathrm{Be}$ and ${ }^{17} \mathrm{C}$ secondary beams on a ${ }^{9} \mathrm{Be}$ target. Section $\mathrm{V}$ contains our concluding remarks.

\section{DEFORMED-CORE EIKONAL FORMULATION}

Single-nucleon knockout reactions are considered where only the projectile residue (but not its final state) is detected following removal of a neutron from the projectile. The two incoherent reaction mechanisms involved are then neutron stripping, the neutron exciting the target, and diffractive breakup of the projectile, the target remaining in its ground state. We use a coordinate system in which the $z$ axis is parallel to the incident projectile beam direction (Fig. 1).

We use the eikonal reaction model, the first step of which is to make an adiabatic treatment [9] of the internal degrees of freedom of the projectile. In the more usual treatment, of a two-body projectile with a spherical core, the internal degrees 


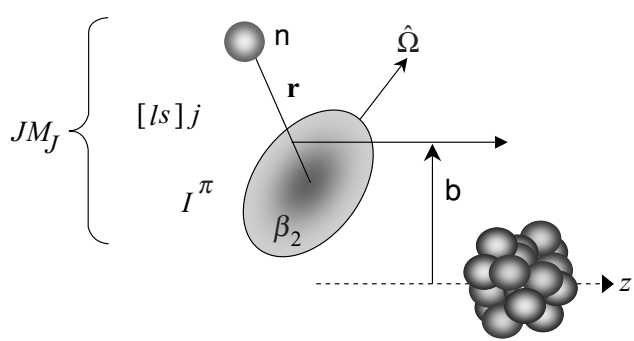

FIG. 1. Schematic of the deformed-core plus neutron projectile system incident on a spherical target indicating the angular momentum quantum numbers, the core orientation $\widehat{\boldsymbol{\Omega}}$, and the center of mass impact parameter $b$.

of freedom are the position coordinate vector $\boldsymbol{r}$ of the removed neutron relative to the core and the internal state of the core. These are assumed fixed for the (short) duration of the (fast) collision between the projectile with the target. In the present deformed core case, both $\boldsymbol{r}$ and the orientation of the deformed core, $\widehat{\boldsymbol{\Omega}}$, are assumed frozen. The model is nonperturbative and treats dissociation of the projectile and excitation, deexcitation, and reorientation of the core degrees of freedom to all orders. The application of this frozen-orientation approximation to calculations of the more restricted problem, of deformed coretarget elastic and inelastic scattering and to calculations of reaction cross sections, can be found in Refs. [10,11].

We thus calculate the cross sections for neutron removal and the population of all final states of the assumed rotational band of the core. Although, at the fragmentation beam energies of interest, these cross sections are expected be dominated by the cross sections to the lowest $0^{+}$and $2^{+}$core final states [10], the model makes no such restriction.

The model system is represented in Fig. 1. The core orientation is described by the spherical polar angles $\widehat{\boldsymbol{\Omega}}=$ $(\alpha, \beta)$ of its symmetry axis. The position of the neutron relative to the center-of-mass (c.m.) of the core, of mass $A_{c}$, is expressed in terms of its cylindrical polar coordinates $\boldsymbol{r}=\left(\boldsymbol{b}_{r}, z_{r}\right) \equiv\left(b_{r}, \phi_{r}, z_{r}\right)$ with $r=\sqrt{b_{r}^{2}+z_{r}^{2}}$. Similarly, the positions of the center-of-mass of the core and the neutron relative to the target are $\boldsymbol{R}_{i}=\left(\boldsymbol{b}_{i}, z_{i}\right) \equiv\left(b_{i}, \phi_{i}, z_{i}\right), i=c, n$, with $b_{c}$ and $b_{n}$ the core and neutron-target impact parameters. The position of the center-of-mass of the composite projectile relative to the target is $\boldsymbol{R}=(\boldsymbol{b}, Z)=(b, \phi, Z)$ and hence

$$
\boldsymbol{b}_{n}=\boldsymbol{b}+\frac{A_{c}}{A_{c}+1} \boldsymbol{b}_{r}, \quad \boldsymbol{b}_{c}=\boldsymbol{b}-\frac{1}{A_{c}+1} \boldsymbol{b}_{r} .
$$

We can take the coordinate $x$ axis in the direction of $\boldsymbol{b}$, thus $\phi=0$.

\section{A. Eikonal model cross sections}

We formulate the neutron removal cross section within the eikonal model. The probability that the neutron survives the collision with the target at an impact parameter $b_{n}$, in the elastic channel, is given by $\left|S_{n}\left(b_{n}\right)\right|^{2}$, the square modulus of its elastic $S$ matrix describing the interaction with the target. Its absorption probability is therefore $1-\left|S_{n}\left(b_{n}\right)\right|^{2}$. Similarly, the probability of the deformed core surviving the collision at an impact parameter $\boldsymbol{b}_{c}$ with orientation $\widehat{\boldsymbol{\Omega}}$ is $\left|S_{c}\left(\boldsymbol{b}_{c}, \widehat{\boldsymbol{\Omega}}\right)\right|^{2}$. The deformation of the core now means that this $S$ matrix is a function of $b_{c}$ and $\hat{\boldsymbol{b}}_{c} \cdot \widehat{\boldsymbol{\Omega}}$ and will couple the states $\Phi_{\operatorname{Im}_{I}}(\widehat{\boldsymbol{\Omega}})$ of the core.

We denote by $\Psi_{J m_{J}}(\boldsymbol{r}, \widehat{\boldsymbol{\Omega}})$ the ground-state wave function of the projectile that describes a deformed core plus loosely bound neutron system, with total angular momentum $J$ and projection $m_{J}$. Generalizing Refs. [3,4] to the deformed core case, the integrated cross section $\sigma_{\text {strip }}$ for single-neutron stripping is now the following:

$$
\begin{aligned}
\sigma_{\text {strip }}= & \frac{1}{2 J+1} \int d \boldsymbol{b} \sum_{m_{J}}\left\langle\Psi_{J m_{J}}(\mathbf{r}, \widehat{\boldsymbol{\Omega}})\right| \\
& \mathcal{O}_{1}\left(\boldsymbol{b}, \boldsymbol{b}_{r}, \widehat{\boldsymbol{\Omega}}\right)\left|\Psi_{J m_{J}}(\boldsymbol{r}, \widehat{\boldsymbol{\Omega}})\right\rangle,
\end{aligned}
$$

where we have introduced the stripping operator

$$
\mathcal{O}_{1}\left(\boldsymbol{b}, \boldsymbol{b}_{r}, \widehat{\boldsymbol{\Omega}}\right)=\left(1-\left|S_{n}\left(b_{n}\right)\right|^{2}\right)\left|S_{c}\left(\boldsymbol{b}_{c}, \widehat{\boldsymbol{\Omega}}\right)\right|^{2}
$$

and where the bra-ket notation on the right-hand side of Eq. (2) implies integration over all $\boldsymbol{r}$, all orientations $\widehat{\boldsymbol{\Omega}}$, and spin variables. Denoting by $\langle\ldots\rangle_{\text {spin }}$ the integration over only the spin variables, we can rewrite this cross section as follows:

$$
\begin{aligned}
\sigma_{\text {strip }}= & \frac{1}{2 J+1} \int d \boldsymbol{b} \int d \boldsymbol{r} \int d \widehat{\boldsymbol{\Omega}} \mathcal{O}_{1}\left(\boldsymbol{b}, \boldsymbol{b}_{r}, \widehat{\boldsymbol{\Omega}}\right) \\
& \times \sum_{m_{J}}\left\langle\left|\Psi_{J_{m_{J}}}(\boldsymbol{r}, \widehat{\boldsymbol{\Omega}})\right|^{2}\right\rangle_{\text {spin }}
\end{aligned}
$$

where we have also assumed that the $S$ matrices, and hence $\mathcal{O}_{1}$, have no spin dependence.

Similarly, the integrated cross section for diffractive or elastic breakup, $\sigma_{\text {diff }}$, in this deformed core plus neutron system is given by the following:

$$
\begin{aligned}
\sigma_{\mathrm{diff}}= & \frac{1}{2 J+1} \int d \boldsymbol{b} \int d \boldsymbol{k} \sum_{m_{J} v} \mid\left\langle\Psi_{v}(\boldsymbol{k})\right| \\
& {\left.\left[1-S_{n}\left(b_{n}\right) S_{c}\right]\left(\boldsymbol{b}_{c}, \widehat{\boldsymbol{\Omega}}\right)\left|\Psi_{J m_{J}}(\boldsymbol{r}, \widehat{\boldsymbol{\Omega}})\right\rangle\right|^{2}, }
\end{aligned}
$$

where the $\Psi_{v}(\boldsymbol{k})$ are the core-plus-neutron unbound states with appropriate neutron and core asymptotic quantum numbers $v$ and wave number $\boldsymbol{k}$. As is usual, the sum over all unbound states can be carried out implicitly using the closure relation. Here we assume the projectile has only one bound state, the ground state, in which case

$$
\begin{aligned}
& \int d \boldsymbol{k} \sum_{v}\left|\Psi_{v}(\boldsymbol{k})\right\rangle\left\langle\Psi_{v}(\boldsymbol{k})\right| \\
& =1-\sum_{m_{J}^{\prime}}\left|\Psi_{J m_{J}^{\prime}}(\boldsymbol{r}, \widehat{\boldsymbol{\Omega}})\right\rangle\left\langle\Psi_{J m_{J}^{\prime}}(\boldsymbol{r}, \widehat{\boldsymbol{\Omega}})\right| .
\end{aligned}
$$

Upon rearrangement of Eq. (5),

$$
\sigma_{\mathrm{diff}}=\frac{1}{2 J+1} \int d \boldsymbol{b}\left[\sum_{m_{J}}\left\langle\Psi_{J m_{J}}(\boldsymbol{r}, \widehat{\boldsymbol{\Omega}})\left|\mathcal{O}_{2}\left(\boldsymbol{b}, \boldsymbol{b}_{r}, \widehat{\boldsymbol{\Omega}}\right)\right| \Psi_{J m_{J}}(\boldsymbol{r}, \widehat{\boldsymbol{\Omega}})\right\rangle-\sum_{m_{J} m_{J}^{\prime}} \mid\left\langle\left.\Psi_{J m_{J}^{\prime}}(\boldsymbol{r}, \widehat{\boldsymbol{\Omega}})\left|\mathcal{O}_{3}\left(\boldsymbol{b}, \boldsymbol{b}_{r}, \widehat{\boldsymbol{\Omega}}\right)\right| \Psi_{J m_{J}}(\boldsymbol{r}, \widehat{\boldsymbol{\Omega}})\right|^{2}\right],\right.
$$


where we have introduced the diffraction mechanism operators

$$
\begin{aligned}
& \mathcal{O}_{2}\left(\boldsymbol{b}, \boldsymbol{b}_{r}, \widehat{\boldsymbol{\Omega}}\right)=\left|\mathcal{O}_{3}\left(\boldsymbol{b}, \boldsymbol{b}_{r}, \widehat{\boldsymbol{\Omega}}\right)\right|^{2}, \\
& \mathcal{O}_{3}\left(\boldsymbol{b}, \boldsymbol{b}_{r}, \widehat{\boldsymbol{\Omega}}\right)=1-S_{n}\left(b_{n}\right) S_{c}\left(\boldsymbol{b}_{c}, \widehat{\boldsymbol{\Omega}}\right) .
\end{aligned}
$$

Expanding once again the bra-ket notation, then

$$
\begin{aligned}
\sigma_{\mathrm{diff}}= & \frac{1}{2 J+1} \int d \boldsymbol{b}\left[\int d \boldsymbol{r} \int d \widehat{\boldsymbol{\Omega}} \mathcal{O}_{2}\left(\boldsymbol{b}, \boldsymbol{b}_{r}, \widehat{\boldsymbol{\Omega}}\right)\right. \\
& \left.\left.\sum_{m_{J}}|| \Psi_{J m_{J}}(\boldsymbol{r}, \widehat{\boldsymbol{\Omega}})\right|^{2}\right\rangle_{\mathrm{spin}}-\sum_{m_{J} m_{J}^{\prime}} \mid \int d \boldsymbol{r} \int d \widehat{\boldsymbol{\Omega}} \\
& \left.\left.\mathcal{O}_{3}\left(\boldsymbol{b}, \boldsymbol{b}_{r}, \widehat{\boldsymbol{\Omega}}\right)\left\langle\Psi_{J m_{J}^{\prime}}(\boldsymbol{r}, \widehat{\boldsymbol{\Omega}}) \mid \Psi_{J m_{J}}(\boldsymbol{r}, \widehat{\boldsymbol{\Omega}})\right\rangle_{\mathrm{spin}}\right|^{2}\right] .
\end{aligned}
$$

We compute Eqs. (4) and (10) for the neutron removal cross sections for this system. The integrals over $\boldsymbol{r}$, the coordinates $z_{r}, b_{r}$, and $\phi_{r}$, and over the angles $\alpha$ and $\beta$ of $\widehat{\boldsymbol{\Omega}}$ will be carried out using numerical quadratures. Having done so, the integral over $\boldsymbol{b}$ requires only the radial c.m. impact parameter $b$ integral because all angular dependence has then been removed.

\section{B. Deformed-core projectile wave functions}

Most generally, the projectile ground-state wave function $\Psi_{J m_{J}}(\boldsymbol{r}, \widehat{\boldsymbol{\Omega}})$ is taken to be a coupled channels eigenstate of a quadrupole-deformed core plus neutron system with total angular momentum $J$ (as in, e.g., Ref [8]). The neutron-core interaction is $\mathcal{V}_{\mathrm{nc}}(\boldsymbol{r}, \widehat{\boldsymbol{\Omega}})$. The orbital angular momentum $\ell$ of the neutron (Fig. 1) is not a good quantum number and is coupled to the intrinsic spin $s$ of the neutron to give $j$ and with the core spin $I$ to give $J$, with an assumed coupling order of $[[\ell \otimes s] j \otimes I] J m_{J}$. The neutron spin $s=1 / 2$ is understood throughout. The neutron spin states are denoted by the spinors $\mathcal{X}_{s m_{s}}$ and the normalized core states by $\Phi_{I m_{I}}(\widehat{\boldsymbol{\Omega}})$. The $\Phi_{I m_{I}}(\widehat{\boldsymbol{\Omega}})$ are assumed to be the states of the $K=0$ rotational band of a rigid rotor, thus $\Phi_{I m_{I}}(\widehat{\boldsymbol{\Omega}}) \equiv Y_{I m_{I}}(\widehat{\boldsymbol{\Omega}})$ and the values of $I$ are restricted to even values.

We thus construct a set of total angular momentum eigenstates

$$
\begin{aligned}
\mathcal{Y}_{\Delta J m_{J}}(\hat{\boldsymbol{r}}, \widehat{\boldsymbol{\Omega}})= & \sum_{m_{\ell} m_{s} m_{j} m_{I}}\left(\ell m_{\ell} s m_{s} \mid j m_{j}\right) \\
& \times\left(j m_{j} I m_{I} \mid J m_{J}\right) \Phi_{I m_{I}}(\widehat{\boldsymbol{\Omega}}) Y_{\ell m_{\ell}}(\hat{\boldsymbol{r}}) \mathcal{X}_{s m_{s}},
\end{aligned}
$$

where $\Delta \equiv(\ell, j, I)$, in which to expand the projectile's ground state as follows:

$$
\Psi_{J m_{J}}(\boldsymbol{r}, \widehat{\boldsymbol{\Omega}})=\sum_{\Delta} R_{\Delta J}(r) \mathcal{Y}_{\Delta J m_{J}}(\hat{\boldsymbol{r}}, \widehat{\boldsymbol{\Omega}}),
$$

in states with parity $(-1)^{\ell}$ and with radial wave functions $R_{\Delta J}(r)$. These radial wave functions were calculated by solving numerically the coupled equations set

$$
\begin{gathered}
\left\{-\frac{\hbar^{2}}{2 \mu}\left[\frac{d^{2}}{d r^{2}}-\frac{\ell(\ell+1)}{r^{2}}\right]+\epsilon_{I}-E_{J}\right\} \mathcal{R}_{\Delta J}(r) \\
=-\sum_{\Delta^{\prime}} \mathcal{V}_{\Delta^{\prime} \Delta}^{J}(r) \mathcal{R}_{\Delta^{\prime} J}(r)
\end{gathered}
$$

with $\mathcal{R}_{\Delta J}(r)=r R_{\Delta J}(r)$ and where the radial coupling potentials are

$$
\mathcal{V}_{\Delta^{\prime} \Delta}^{J}(r)=\left\langle\mathcal{Y}_{\Delta^{\prime} J m_{J}}(\hat{\boldsymbol{r}}, \widehat{\boldsymbol{\Omega}})\left|\mathcal{V}_{\mathrm{nc}}(\boldsymbol{r}, \widehat{\boldsymbol{\Omega}})\right| \mathcal{Y}_{\Delta_{J} m_{J}}(\hat{\boldsymbol{r}}, \widehat{\boldsymbol{\Omega}})\right\rangle,
$$

$\mu$ is the reduced mass of the neutron-core system and $\epsilon_{I}$ is the energy of core state $I$.

Following Ref. [8], the coupled channels equations are solved assuming $\mathcal{V}_{\mathrm{nc}}$ is the sum of a deformed Woods-Saxon potential $V_{\mathrm{ws}}(\boldsymbol{r}, \widehat{\boldsymbol{\Omega}})$ plus a spherical spin-orbit term $V_{\mathrm{so}}(r)$. The deformed Woods-Saxon potential is as follows:

$$
V_{\mathrm{ws}}(\boldsymbol{r}, \widehat{\boldsymbol{\Omega}})=-V_{\mathrm{ws}} /\left\{1+\exp \left[(r-R(\hat{\boldsymbol{r}} \cdot \widehat{\boldsymbol{\Omega}})) / a_{\mathrm{ws}}\right]\right\}
$$

and has strength $V_{\mathrm{ws}}$, diffuseness $a_{\mathrm{ws}}$, and deformed core radius parameter

$$
R(\hat{\boldsymbol{r}} \cdot \widehat{\boldsymbol{\Omega}})=R_{\mathrm{ws}}\left[1+\beta_{2} Y_{20}(\hat{\boldsymbol{r}} \cdot \widehat{\boldsymbol{\Omega}})\right]
$$

with quadrupole deformation $\beta_{2}$. The spin-orbit term is, in standard notation,

$$
V_{\text {so }}(r)=2 V_{\text {so }}\left(\frac{\hbar}{m_{\pi} c}\right)^{2} \frac{1}{r} \frac{d}{d r}\left[1+\exp \left(\frac{r-R_{\mathrm{so}}}{a_{\mathrm{so}}}\right)\right]^{-1} \boldsymbol{\ell} \cdot \boldsymbol{s} .
$$

The potentials are defined such that their strengths $V_{\text {ws }}$ and $V_{\text {so }}$ are positive.

\section{Reduction of structure terms}

To use Eqs. (4) and (10) we require the spin variable integral of the general wave function product $\mathcal{I}_{m_{J}^{\prime} m_{J}}^{J}=$ $\left\langle\Psi_{J m_{J}^{\prime}}(\boldsymbol{r}, \widehat{\boldsymbol{\Omega}}) \mid \Psi_{J m_{J}}(\boldsymbol{r}, \widehat{\boldsymbol{\Omega}})\right\rangle_{\text {spin }}$. This can be simplified using the explicit forms of Eqs. (11) and (12). It follows from Eq. (12) that

$$
\begin{aligned}
\mathcal{I}_{m_{J}^{\prime} m_{J}}^{J}= & \sum\left(\ell^{\prime} m_{\ell^{\prime}} s m_{s} \mid j^{\prime} m_{j^{\prime}}\right)\left(j^{\prime} m_{j^{\prime}} I^{\prime} m_{I^{\prime}} \mid J m_{J}^{\prime}\right) \\
& \times\left(\ell m_{\ell} s m_{s} \mid j m_{j}\right)\left(j m_{j} I m_{I} \mid J m_{J}\right) Y_{I^{\prime} m_{I^{\prime}}}^{*}(\widehat{\boldsymbol{\Omega}}) \\
& \times Y_{I m_{I}}(\widehat{\boldsymbol{\Omega}}) Y_{\ell^{\prime} m_{\ell^{\prime}}}^{*}(\hat{\boldsymbol{r}}) Y_{\ell m_{\ell}}(\hat{\boldsymbol{r}}) R_{\left(\ell^{\prime} s j^{\prime} I^{\prime}\right) J}(r) R_{(\ell s j I) J}(r),
\end{aligned}
$$

summed over $\ell, \ell^{\prime}, j, j^{\prime}, I, I^{\prime}, m_{\ell}, m_{\ell^{\prime}}, m_{j}, m_{j^{\prime}}, m_{I}, m_{I^{\prime}}$, and $m_{s}$. On recoupling each pair of spherical harmonics, $[\ell \otimes$ $\left.\ell^{\prime}\right] \mathcal{L} m_{\mathcal{L}}$ and $\left[I \otimes I^{\prime}\right] \mathcal{I} m_{\mathcal{I}}$, using Ref. [12], then

$$
\begin{aligned}
Y_{\ell^{\prime} m_{\ell^{\prime}}}^{*}(\hat{\boldsymbol{r}}) Y_{\ell m_{\ell}}(\hat{\boldsymbol{r}})= & \sum_{\mathcal{L} m_{\mathcal{L}}}(-1)^{m_{\ell^{\prime}}} \frac{\hat{\ell} \hat{\ell}^{\prime}}{\sqrt{4 \pi} \hat{\mathcal{L}}}\left(\ell 0 \ell^{\prime} 0 \mid \mathcal{L} 0\right) \\
& \times\left(\ell m_{\ell} \ell^{\prime}-m_{\ell^{\prime}} \mid \mathcal{L} m_{\mathcal{L}}\right) Y_{\mathcal{L} m_{\mathcal{L}}}(\hat{\boldsymbol{r}}),
\end{aligned}
$$

and performing several of the angular momentum projection sums then one obtains the following:

$$
\begin{aligned}
\mathcal{I}_{m_{J}^{\prime} m_{J}}^{J}= & \hat{J} \sum \frac{\hat{\ell} \hat{\ell}^{\prime} \hat{j} \hat{j}^{\prime} \hat{I} \hat{I}^{\prime} \hat{k}}{4 \pi}(-1)^{\xi}\left(I 0 I^{\prime} 0 \mid \mathcal{I} 0\right)\left(\ell 0 \ell^{\prime} 0 \mid \mathcal{L} 0\right) \\
& \times\left(J m_{J} k-m_{k} \mid J m_{J}^{\prime}\right)\left(\mathcal{L} m_{\mathcal{L}} \mathcal{I} m_{\mathcal{I}} \mid k m_{k}\right) \\
& \times W\left(\ell^{\prime} j^{\prime} \ell j ; s \mathcal{L}\right)\left\{\begin{array}{ccc}
I^{\prime} & I & \mathcal{I} \\
j^{\prime} & j & \mathcal{L} \\
J & J & k
\end{array}\right\} Y_{\mathcal{L} m_{\mathcal{L}}(\hat{\boldsymbol{r}}) Y_{\mathcal{I} m_{\mathcal{I}}}(\widehat{\boldsymbol{\Omega}})} \\
& \times R_{(\ell s j I) J}(r) R_{\left(\ell^{\prime} s j^{\prime} I^{\prime}\right) J}(r),
\end{aligned}
$$


where the phase factor is $\xi=2 j^{\prime}+I^{\prime}+m_{k}-s-j$ and the remaining sum is now over $\ell, \ell^{\prime}, j, j^{\prime}, I, I^{\prime}, \mathcal{L}, \mathcal{I}, k, m_{\mathcal{L}}, m_{\mathcal{I}}$, and $m_{k}$.

Further simplifications can be made to the first term in the diffractive cross section, Eq. (10), and the stripping cross section, Eq. (4), which require only $\sum_{m_{J}} \mathcal{I}_{m_{J} m_{J}}^{J}$. This restricts contributions to the sums from those terms with $\mathcal{I}=\mathcal{L}, m_{\mathcal{I}}=$ $-m_{\mathcal{L}}$, and

$$
\begin{aligned}
\sum_{m_{J}} \mathcal{I}_{m_{J} m_{J}}^{J}= & \hat{J}^{2} \sum \frac{\hat{\ell} \hat{\ell^{\prime}} \hat{j} \hat{j}^{\prime} \hat{I} \hat{I}^{\prime}}{4 \pi \hat{\mathcal{L}}^{2}}(-1)^{2 j^{\prime}+J-s-2 j}\left(I 0 I^{\prime} 0 \mid \mathcal{L} 0\right) \\
& \times\left(\ell 0 \ell^{\prime} 0 \mid \mathcal{L} 0\right) W\left(\ell^{\prime} j^{\prime} \ell j ; s \mathcal{L}\right) W\left(I j I^{\prime} j^{\prime} ; J \mathcal{L}\right) \\
& \times Y_{\mathcal{L} m_{\mathcal{L}}}(\hat{\boldsymbol{r}}) Y_{\mathcal{L} m_{\mathcal{L}}}^{*}(\widehat{\boldsymbol{\Omega}}) R_{(\ell s j I) J}(r) R_{\left(\ell^{\prime} s j^{\prime} I^{\prime}\right) J}(r),
\end{aligned}
$$

the sum being over $\ell, \ell^{\prime}, j, j^{\prime}, I, I^{\prime}, \mathcal{L}$, and $m_{\mathcal{L}}$.

\section{Cross section calculations}

The general structure of the spin-integrated wave function product $\left\langle\Psi_{J m_{J}^{\prime}}(\boldsymbol{r}, \widehat{\boldsymbol{\Omega}}) \mid \Psi_{J m_{J}}(\boldsymbol{r}, \widehat{\boldsymbol{\Omega}})\right\rangle_{\text {spin }}$ is given by Eq. (20). To include this in the cross section expressions, Eqs. (4) and (10), we now define the quantities as follows:

$$
\begin{aligned}
Z_{k m_{k}}^{J, q}(b)= & \int d \widehat{\boldsymbol{\Omega}} \int d \boldsymbol{r} \mathcal{O}_{q}\left(\boldsymbol{b}, \boldsymbol{b}_{r}, \widehat{\boldsymbol{\Omega}}\right) \sum_{\mathcal{L} m_{\mathcal{L}} \mathcal{I} m_{\mathcal{I}}}\left(\mathcal{L} m_{\mathcal{L}} \mathcal{I} m_{\mathcal{I}} \mid k m_{k}\right) \\
& \times Y_{\mathcal{L} m_{\mathcal{L}}}(\hat{\boldsymbol{r}}) Y_{\mathcal{I} m_{\mathcal{I}}}(\widehat{\boldsymbol{\Omega}}) T_{\mathcal{L} \mathcal{I} k}^{J}(r),
\end{aligned}
$$

where $T_{\mathcal{L} \mathcal{I} k}^{J}(r)$ takes the form of a transition density and is as follows:

$$
\begin{aligned}
T_{\mathcal{L I k}}^{J}(r)= & \hat{J} \hat{k} \sum_{\ell j I \ell^{\prime} j^{\prime} I^{\prime}} \frac{\hat{\ell} \hat{\ell}^{\prime} \hat{I} \hat{I}^{\prime} \hat{j} \hat{j}^{\prime}}{4 \pi}(-1)^{2 j^{\prime}+I^{\prime}-s-j}\left(I 0 I^{\prime} 0 \mid \mathcal{I} 0\right) \\
& \times\left(\ell 0 \ell^{\prime} 0 \mid \mathcal{L} 0\right) W\left(\ell^{\prime} j^{\prime} \ell j ; s \mathcal{L}\right)\left\{\begin{array}{ccc}
I^{\prime} & I & \mathcal{I} \\
j^{\prime} & j & \mathcal{L} \\
J & J & k
\end{array}\right\} \\
& \times R_{(\ell s j I) J}(r) R_{\left(\ell^{\prime} s j^{\prime} I^{\prime}\right) J}(r) .
\end{aligned}
$$

In terms of these $Z_{k m_{k}}^{J, q}(b)$, it follows that

$$
\sigma_{\text {strip }}=2 \pi \int_{0}^{\infty} d b b Z_{00}^{J, 1}(b)
$$

and

$$
\begin{aligned}
\sigma_{\text {diff }}= & 2 \pi \int_{0}^{\infty} d b b\left[Z_{00}^{J, 2}(b)-\frac{1}{2 J+1} \sum_{m_{J} m_{J}^{\prime}} \mid \sum_{k m_{k}}(-1)^{m_{k}}\right. \\
& \left.\left.\left(J m_{J} k-m_{k} \mid J m_{J}^{\prime}\right) Z_{k m_{k}}^{J, 3}(b)\right|^{2}\right] .
\end{aligned}
$$

Expressing the spherical harmonics in terms of the associated Legendre functions and the integrals over $\widehat{\boldsymbol{\Omega}}$ and $\boldsymbol{r}$ in terms of their spherical and cylindrical coordinates, respectively, the
$Z_{k m_{k}}^{J, q}(b)$ can be written as follows:

$$
\begin{aligned}
Z_{k m_{k}}^{J, q}(b)= & \int_{0}^{2 \pi} d \phi_{r} \int_{0}^{\infty} d b_{r} \int_{-1}^{+1} d \cos \alpha \\
& \times \sum_{\mathcal{L} m_{\mathcal{L}} m_{m_{\mathcal{I}}}}\left(\mathcal{L} m_{\mathcal{L}} \mathcal{I}_{m_{\mathcal{I}}} \mid k m_{k}\right) C_{\mathcal{L}}^{m_{\mathcal{L}}} C_{\mathcal{I}}^{m_{\mathcal{I}}} P_{\mathcal{I}}^{m_{\mathcal{I}}}(\cos \alpha) \\
& \times e^{i m_{\mathcal{L}} \phi_{r}} G_{\mathcal{L} m_{\mathcal{L}} \mathcal{I} k}^{J}\left(b_{r}\right) \int_{0}^{2 \pi} d \beta \\
& \times e^{i m_{\mathcal{I}} \beta} \mathcal{O}_{q}\left(b, b_{r}, \phi_{r}, \beta, \alpha\right)
\end{aligned}
$$

The $C_{\mathcal{L}}^{\mathcal{M}}$ are given by the following:

$$
C_{\mathcal{L}}^{\mathcal{M}}=(-1)^{(\mathcal{M}+|\mathcal{M}|) / 2} \sqrt{\frac{2 \mathcal{L}+1}{4 \pi} \frac{(\mathcal{L}-|\mathcal{M}|) !}{(\mathcal{L}+|\mathcal{M}|) !}}
$$

and the $z$ coordinate integrals are

$$
G_{\mathcal{L} m_{\mathcal{L}} \mathcal{I} k}^{J}\left(b_{r}\right)=b_{r} \int_{-\infty}^{\infty} d z_{r} P_{\mathcal{L}}^{m_{\mathcal{L}}}\left(z_{r} / r\right) T_{\mathcal{L} \mathcal{I} k}^{J}(r) .
$$

The functions $T_{\mathcal{L} \mathcal{I k}}^{J}$ and the neutron-target and the deformed core-target $S$ matrices, $S_{n}\left(b_{n}\right)$ and $S_{c}\left(\boldsymbol{b}_{c}, \widehat{\boldsymbol{\Omega}}\right) \equiv S_{c}\left(b_{c}, \phi_{c}-\right.$ $\beta, \alpha)$, entering the $\mathcal{O}_{q}$, are precalculated over the required range of arguments and are interpolated when needed. The values of $b_{n}, b_{c}$, and $\phi_{c}$ are expressed in terms of $b, b_{r}$, and $\phi_{r}$, as given by Eq. (1). The calculation of the $S$ matrices is discussed in the next section.

Equations (24) and (25) calculate the cross sections for neutron removal and that populate any state $I^{\pi}$ of the assumed rotational band of the core. However, analogous calculations of rotational inelastic scattering at fragmentation beam energies suggest that cross sections will be dominated by the channels involving the lowest $0^{+}$and $2^{+}$states of the core $[6,10]$.

\section{CORE- AND NEUTRON-TARGET $S$ MATRICES}

The deformed core and neutron-target $S$ matrices are calculated within the optical limit of Glauber's multiple scattering theory [9]. These involve a double and single folding, respectively, of the particle and target one-body densities with an effective nucleon-nucleon $(N N)$ interaction. We assume here, for simplicity, a zero-range delta interaction. In the case of a quadrupole deformed core of density $\rho_{c}(\boldsymbol{r}, \widehat{\boldsymbol{\Omega}})$ interacting with a spherical target of density $\rho_{t}(r)$ then

$$
S_{c}\left(\boldsymbol{b}_{c}, \widehat{\boldsymbol{\Omega}}\right)=\exp \left[-\bar{\sigma}_{N N}\left(1-i \bar{\alpha}_{N N}\right) \chi_{c}\left(\boldsymbol{b}_{c}, \widehat{\boldsymbol{\Omega}}\right) / 2\right],
$$

where (e.g., Ref. [11]):

$$
\chi_{c}\left(\boldsymbol{b}_{c}, \widehat{\boldsymbol{\Omega}}\right)=\int_{-\infty}^{\infty} d z_{c} \int d \boldsymbol{r} \rho_{c}(\boldsymbol{r}, \widehat{\boldsymbol{\Omega}}) \rho_{t}\left(\left|\boldsymbol{r}+\boldsymbol{R}_{c}\right|\right) .
$$

Here $\bar{\sigma}_{N N}$ is the average of the free-space $n n$ and $n p$ total cross sections and $\bar{\alpha}_{N N}$ is the appropriate cross-section-weighted average of the real-to-imaginary parts of the forward $N N$ scattering amplitudes (e.g., Ref [13]). We assume a Gaussian target density

$$
\rho_{t}(r)=\rho_{t 0} \exp \left[-\gamma r^{2}\right]
$$


with range fixed by the target root-mean-square (rms) matter radius.

A quadrupole deformed Woods-Saxon form factor is used for the core density

$$
\rho_{c}(\boldsymbol{r}, \widehat{\boldsymbol{\Omega}})=\rho_{c 0} /\left\{1+\exp \left[(r-R(\hat{\boldsymbol{r}} \cdot \widehat{\boldsymbol{\Omega}})) / a_{\mathrm{ws}}\right]\right\} .
$$

Here the core radius, $R(\hat{\boldsymbol{r}} \cdot \widehat{\boldsymbol{\Omega}})$, has a form identical to that in Eq. (16). The corresponding $R_{\mathrm{ws}}$ radius parameter and overall strength $\rho_{c 0}$ are determined, given an assumed $a_{\mathrm{ws}}$ and $\beta_{2}$, to give the nucleon number $A_{c}$ and the rms matter radius of the core nucleus. Following Ref. [11] the phase entering the core $S$ matrix can be expressed as a Legendre polynomial expansion

$$
\chi_{c}\left(\boldsymbol{b}_{c}, \widehat{\boldsymbol{\Omega}}\right)=\sum_{L} \int_{-\infty}^{\infty} d z_{c} \mathcal{Q}_{L}\left(R_{c}\right) P_{L}\left(\hat{\boldsymbol{R}}_{c} \cdot \widehat{\boldsymbol{\Omega}}\right)
$$

where, for the densities used above,

$$
\begin{aligned}
\mathcal{Q}_{L}\left(R_{c}\right)= & 4 \pi \rho_{t 0} \exp \left(-\gamma R_{c}^{2}\right) \int_{0}^{\infty} d r r^{2} \bar{\rho}_{c L}(r) \\
& \times \exp \left(-\gamma r^{2}\right) i^{L} j_{L}\left(2 i \gamma R_{c} r\right) .
\end{aligned}
$$

The $\bar{\rho}_{c L}(r)$ are the multipole components of the deformed Woods-Saxon or Fermi form factor. These are expressed analytically, to order $\beta_{2}^{3}$, in Appendix B of Ref. [10], which are used in the present analysis. We include multipole terms with $L=0,2$, and 4 in Eq. (33).

Because the unit vectors $\hat{\boldsymbol{R}}_{c}$ and $\widehat{\boldsymbol{\Omega}}$ are as follows:

$$
\hat{\boldsymbol{R}}_{c}=\left[b_{c} \cos \phi_{c} \hat{\mathbf{i}}+b_{c} \sin \phi_{c} \hat{\mathbf{j}}+z_{c} \hat{\boldsymbol{k}}\right] / R_{c}
$$

and

$$
\widehat{\boldsymbol{\Omega}}=\sin \alpha \cos \beta \hat{\mathbf{i}}+\sin \alpha \sin \beta \hat{\mathbf{j}}+\cos \alpha \hat{\boldsymbol{k}},
$$

the argument of the Legendre polynomials in Eq. (33) is as follows:

$$
\hat{\boldsymbol{R}}_{c} \cdot \widehat{\boldsymbol{\Omega}}=\left[b_{c} \sin \alpha \cos \left(\phi_{c}-\beta\right)+z_{c} \cos \alpha\right] / R_{c} .
$$

Noting also that all terms in the integrand in Eq. (33) are even functions of $z_{c}$, then

$$
\begin{aligned}
\chi_{c}\left(\boldsymbol{b}_{c}, \widehat{\boldsymbol{\Omega}}\right)= & \chi_{c}\left(b_{c}, \phi_{c}-\beta, \alpha\right) \\
= & \int_{0}^{\infty} d z_{c} \mathcal{Z}_{1}\left(R_{c}, \alpha\right)+\cos ^{2}\left(\phi_{c}-\beta\right) \int_{0}^{\infty} d z_{c} \\
& \times \mathcal{Z}_{2}\left(R_{c}, \alpha\right)+\cos ^{4}\left(\phi_{c}-\beta\right) \int_{0}^{\infty} d z_{c} \mathcal{Z}_{3}\left(R_{c}, \alpha\right)
\end{aligned}
$$

with

$$
\begin{aligned}
\mathcal{Z}_{1}\left(R_{c}, \alpha\right)= & 2 \mathcal{Q}_{0}\left(R_{c}\right)+\frac{\left[3 z_{c}^{2} \cos ^{2} \alpha-R_{c}^{2}\right]}{R_{c}^{2}} \mathcal{Q}_{2}(r) \\
& -\frac{\left[30 z_{c}^{2} \cos ^{2} \alpha-3 R_{c}^{2}\right]}{4 R_{c}^{2}} \mathcal{Q}_{4}\left(R_{c}\right) \\
& +\frac{35}{4} \frac{z_{c}^{4} \cos ^{4} \alpha}{R_{c}^{4}} \mathcal{Q}_{4}\left(R_{c}\right),
\end{aligned}
$$

$$
\begin{aligned}
\mathcal{Z}_{2}\left(R_{c}, \alpha\right)= & \frac{3 b_{c}^{2} \sin ^{2} \alpha}{R_{c}^{2}} \mathcal{Q}_{2}\left(R_{c}\right)-\frac{15}{2} \frac{b_{c}^{2} \sin ^{2} \alpha}{R_{c}^{2}} \mathcal{Q}_{4}\left(R_{c}\right) \\
& +\frac{105}{2} \frac{z_{c}^{2} b_{c}^{2} \sin ^{2} \alpha \cos ^{2} \alpha}{R_{c}^{4}} \mathcal{Q}_{4}\left(R_{c}\right) \\
\mathcal{Z}_{3}\left(R_{c}, \alpha\right)= & \frac{35}{4} \frac{b_{c}^{4} \sin ^{4} \alpha}{R_{c}^{4}} \mathcal{Q}_{4}\left(R_{c}\right) .
\end{aligned}
$$

The integrals of these $\mathcal{Z}_{i}$ over $z_{c}$ in Eq. (38) can be calculated and stored as an efficient way to evaluate the $S_{c}\left(\boldsymbol{b}_{c}, \widehat{\boldsymbol{\Omega}}\right)$.

The neutron $S$ matrix, by comparison, is calculated more simply, using

$$
S_{n}\left(b_{n}\right)=\exp \left[-\bar{\sigma}_{N N}\left(1-i \bar{\alpha}_{N N}\right) \chi_{n}\left(b_{n}\right) / 2\right],
$$

where

$$
\chi_{n}\left(b_{n}\right)=\int_{-\infty}^{\infty} d z_{n} \rho_{t}\left(R_{n}\right)
$$

\section{APPLICATIONS TO SINGLE-NEUTRON KNOCKOUT}

We now apply the formalism, developed in detail in Secs. II and III, to model two specific knockout reaction systems. Both chosen reactions were the subject of recent experiments carried out at the National Superconducting Cyclotron Laboratory at Michigan State University. These are the ${ }^{9} \mathrm{Be}\left({ }^{11} \mathrm{Be},{ }^{10} \mathrm{Be}\right) \mathrm{X}$ reaction at a beam energy of $60 \mathrm{MeV}$ per nucleon [6] and the ${ }^{9} \mathrm{Be}\left({ }^{17} \mathrm{C},{ }^{16} \mathrm{C}\right) \mathrm{X}$ reaction at $62 \mathrm{MeV}$ per nucleon [14]. The projectile ground-states are $1 / 2^{+}$and $3 / 2^{+}$, respectively. These examples have been chosen as there is evidence of significant neutron plus excited core components in the ${ }^{11} \mathrm{Be}$ and ${ }^{17} \mathrm{C}$ ground-states and their low-energy structures. There were also discrepancies noted between the spherical spectator core calculations and the data in both instances $[6,14]$.

\section{A. Application to ${ }^{11} \mathrm{Be}$}

The $J=1 / 2,{ }^{11} \mathrm{Be}$ projectile ground state has positive parity and, when including $I^{\pi}=0^{+}$and $2^{+}$core states, has ground-state configurations $\left[n \ell_{j} \otimes I^{\pi}\right] J$ with orbital angular momenta $\ell=0$ and 2. Our wave function, Eq. (12), is a superposition of $\left[2 s_{1 / 2} \otimes 0^{+}\right],\left[1 d_{3 / 2} \otimes 2^{+}\right]$, and $\left[1 d_{5 / 2} \otimes 2^{+}\right]$ neutron configurations [8] with spectroscopic factors of 0.85 , 0.02 , and 0.13 , respectively. The radial wave functions were calculated by numerically solving the coupled channels set, Eq. (13). We use a deformation parameter $\beta_{2}=+0.67$ computed [8] from the $B(E 2)$ value of Ref. [15] and the ${ }^{10} \mathrm{Be}$ $2^{+}$state energy is $\epsilon_{2}=3.4 \mathrm{MeV}$. The neutron-core potential parameters used were $V_{\mathrm{ws}}=54.24 \mathrm{MeV}, V_{\text {so }}=8.50 \mathrm{MeV}$, $a_{\mathrm{ws}}=a_{\mathrm{so}}=0.65 \mathrm{fm}$, and $R_{\mathrm{ws}}=R_{\mathrm{so}}=2.483 \mathrm{fm}$. With these parameters the coupled channels ground state reproduces the ${ }^{11} \mathrm{Be}$ neutron separation energy of $0.5 \mathrm{MeV}$. Because $J=1 / 2$, there are contributions to the cross sections only from $k=0$ and hence $\mathcal{I}=\mathcal{L}=0,2$, 4 in Eq. (25).

Following Sec. III, the calculation of the core-target $S$ matrix uses a Woods-Saxon ${ }^{10}$ Be matter density with $\mathrm{rms}$ radius of $2.28 \mathrm{fm}$ [16], $\beta_{2}=+0.67$ and a diffuseness of $0.5 \mathrm{fm}$. For the ${ }^{9} \mathrm{Be}$ target we used a spherical Gaussian density 
with rms matter radius of $2.36 \mathrm{fm}$ [17]. The value of $\bar{\sigma}_{N N}$, calculated from the free $n p$ and $n n$ total cross sections using the formulas of Ref. [18], was $88.0 \mathrm{mb}$. The $\bar{\alpha}_{N N}$ value was then computed from the $100 \mathrm{MeV}$ real to imaginary $n n$ and $n p$ amplitude ratios, 1.87 and 1.00 of Ref. [13]. The resulting value is $\bar{\alpha}_{N N}=1.22$. To assess the core deformation effects on the reaction dynamics, calculations using this $S_{c}\left(\boldsymbol{b}_{c}, \widehat{\boldsymbol{\Omega}}\right)$ are compared with the corresponding (spherical core) calculations using the $S_{c}\left(b_{c}\right)$ computed assuming $\beta_{2}=0$ and with the same core rms matter radius, $2.28 \mathrm{fm}$.

With $\beta_{2}=0.67$ the full calculations yield $\sigma_{\text {strip }}=108.9 \mathrm{mb}$ and $\sigma_{\text {diff }}=86.5 \mathrm{mb}$. With the spherical $S_{c}, \sigma_{\text {strip }}=108.3 \mathrm{mb}$ and $\sigma_{\text {diff }}=78.3 \mathrm{mb}$. So, upon including the deformed-core $S$ matrix the inclusive diffractive cross section is increased by $8.2 \mathrm{mb}$, which, given the large $0^{+}$state spectroscopic factor of 0.85 , is expected to feed predominantly the $2^{+}$core state. These fully dynamical calculations are therefore consistent with the earlier, magnitude estimate of $8 \mathrm{mb}$ (for an assumed $0^{+}$state spectroscopic factor of 0.74) made in Ref. [6]. The stripping cross section is seen to be essentially unchanged within our weak-coupling approach, consistent with the findings of the strong-coupling stripping calculations of Ref. [7].

Our ability to compare our model calculations with the experimental data is hindered by their inclusive nature. To make such a comparison, the present model calculations are most realistically compared with the sum of the measured partial cross sections to the $0^{+}$[203(31) mb] and $2^{+}$[16(4) mb] states in ${ }^{10} \mathrm{Be}$. There is also measured cross section to negative parity states in ${ }^{10} \mathrm{Be}$, at around $6 \mathrm{MeV}$, but this is the result of neutron removal from the ${ }^{10} \mathrm{Be}$ core and such effects are absent from our present two-body model. Our full dynamical calculation yields an inclusive $0^{+}$and $2^{+}$state single neutron removal cross section of $195 \mathrm{mb}$. This compares well with the measured value of 219(31) $\mathrm{mb}$ [6].

\section{B. Application to ${ }^{17} \mathrm{C}$}

Another very interesting reaction system is the removal of a single (weakly bound) neutron from ${ }^{17} \mathrm{C}$. The ground-state-toground-state neutron separation energy is $0.729 \mathrm{MeV}$. However, the shell model predicts only a very small component of the ${ }^{16} \mathrm{C}\left(0^{+}\right)$ground state configuration in the ${ }^{17} \mathrm{C}\left(3 / 2^{+}\right)$ground state, with a spectroscopic factor of only 0.03 [14]. Thus, within a conventional spherical spectator-core calculation, this theoretical $\ell=2$ ground-state-to-ground-state neutron knockout partial cross section is only $2 \mathrm{mb}$. Experimentally, however, this partial cross section is measured to be 22(11) $\mathrm{mb}$ [14]. The shell model suggests that the major ${ }^{17} \mathrm{C}$ groundstate spectroscopic strength (of 1.44) is associated with an $\ell=2$ neutron coupled to the ${ }^{16} \mathrm{C}\left(2^{+}\right)$core excited state with $E^{*}=1.77 \mathrm{MeV}$ and thus with neutron separation energy of $2.5 \mathrm{MeV}$.

Here we model the ${ }^{17} \mathrm{C}\left(J=3 / 2^{+}\right)$ground state by use of our quadrupole-deformed-core plus neutron model. As there is vanishing shell model strength to the ${ }^{16} \mathrm{C}\left(0^{+}\right)$core state, the projectile wave function is described as a pure $\left[1 d_{5 / 2} \otimes 2^{+}\right] J$ configuration, where the single particle radial wave function $R_{(\ell s j I) J}(r)$ is calculated in a central Woods-Saxon potential $\left(V_{\mathrm{so}}=0, \beta_{2}=0\right)$ with radius parameter $R_{\mathrm{ws}}=1.25 A_{c}^{1 / 3} \mathrm{fm}$

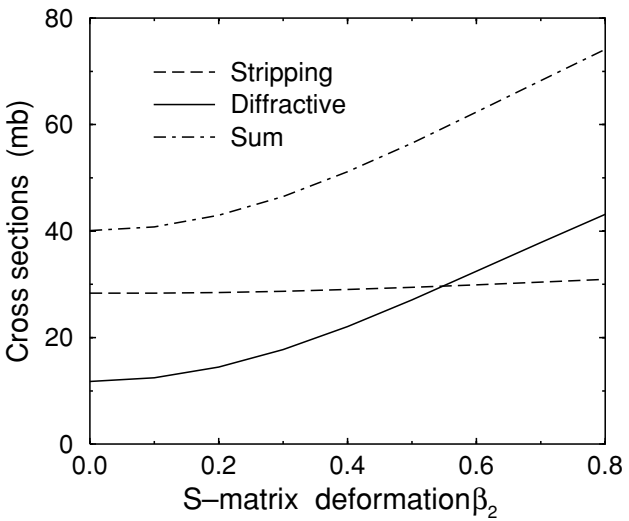

FIG. 2. Dependence of the calculated stripping and diffractive single-neutron removal cross sections, and their sum, upon the assumed core deformation $\beta_{2}$ for the ${ }^{9} \mathrm{Be}\left({ }^{17} \mathrm{C},{ }^{16} \mathrm{C}\right) \mathrm{X}$ reaction at $62 \mathrm{MeV}$ per nucleon. The calculations are for a spectroscopic factor of 1 .

and diffuseness $a_{\mathrm{ws}}=0.7 \mathrm{fm}$. For the calculations of the deformed core-target $S$ matrix we used a Woods-Saxon ${ }^{16} \mathrm{C}$ matter density with rms radius of $2.70 \mathrm{fm}$ [17], a diffuseness of $0.5 \mathrm{fm}$, and deformation $\beta_{2}$. The choice of this deformation is discussed below. The Gaussian ${ }^{9} \mathrm{Be}$ target density was the same as used in the ${ }^{11} \mathrm{Be}$ projectile case, above. The value of $\bar{\sigma}_{N N}$ was now $84.7 \mathrm{mb}$ and, from the $100 \mathrm{MeV}$ real to imaginary $n n$ and $n p$ amplitude ratios, 1.87 and 1.00 of [13], $\bar{\alpha}_{N N}=1.23$.

In this ${ }^{17} \mathrm{C}$ case, because of our assumed fully $2^{+}$core ground state, the core deformation $\beta_{2}$ enters only into the deformed core-target $S$ matrix. The choice of a realistic ${ }^{16} \mathrm{C}$ core deformation is less clear. We note, for example, the recent measurement by Imai et al. [19] of an anomalously small $B\left(E 2: 2_{1}^{+} \rightarrow 0^{+}\right)$strength in ${ }^{16} \mathrm{C}$, with implications for details of both the core structure and for its charge deformation. Because, in the present application, core excitation is entirely macroscopic and strong interaction induced, because of $S_{c}\left(\boldsymbol{b}_{c}, \widehat{\boldsymbol{\Omega}}\right)$, further detailed consideration of this core structure is quite beyond the scope of the present model, where it enters only through a mass $\beta_{2}$. We thus comment below on the dependence of the calculated cross sections on a wide range of assumed $\beta_{2}$ values.

For such an orientation, we first assume a maximal mass deformation, $\beta_{2}=0.55$, typical of values extracted from analyses of data from (surface dominated) light-ion and heavy-ion induced inelastic scattering of ${ }^{12} \mathrm{C}$ [20,21]. For $\beta_{2}=0.55$ the calculated single particle cross sections (for unit spectroscopic factor) are $\sigma_{\text {strip }}=29.7 \mathrm{mb}$ and $\sigma_{\text {diff }}=$ $29.7 \mathrm{mb}$. When using the spherical $S_{c}, \sigma_{\text {strip }}=28.3 \mathrm{mb}$ and $\sigma_{\text {diff }}=11.8 \mathrm{mb}$. So, when including this maximally deformedcore $S$ matrix the inclusive diffractive cross section is increased by $17.9 \mathrm{mb}$. Given that, in our model, we have incident flux only in the $2^{+}$core state channel, this enhanced diffractive cross section may be expected to feed, predominantly, the ${ }^{16} \mathrm{C}$ $0^{+}$state. Once again, the stripping cross section is found to be essentially unchanged by the core dynamics, consistent with Ref. [7]. The dependence of our fully dynamical model $\sigma_{\text {diff }}$ and $\sigma_{\text {strip }}$ on the assumed $\beta_{2}$ value are shown in Fig. 2. The 
calculations presented are the single-particle cross sections calculated with unit spectroscopic factor. Compared to the spherical core value $\sigma_{\text {diff }}=11.8 \mathrm{mb}$, the calculated values are $14.5 \mathrm{mb}$ and $22.1 \mathrm{mb}$, respectively, for $\beta_{2}=0.20$ and 0.40 , $\sigma_{\text {diff }}$ increasing monotonically with deformation.

Our model calculations may be compared with the sum of the measured partial cross sections to the $0^{+}[22(11) \mathrm{mb}]$ and $2^{+}[60(12) \mathrm{mb}]$ states of ${ }^{16} \mathrm{C}$, namely $82(16) \mathrm{mb}$ [14]. There is also measured strength to the $4^{+}$state near $4 \mathrm{MeV}$ of excitation, but this is not resolved from neighboring $J=2$ and $J=3$ states and is not quantified here. When including the $\left[1 d_{5 / 2} \otimes 2^{+}\right]$state shell model spectroscopic factor of 1.44 we obtain a theoretical cross section of $\sigma_{\text {th }}=86 \mathrm{mb}$ from the maximally deformed case (with $\beta_{2}=0.55$ ) and $58 \mathrm{mb}$ from the spherical core calculation. We can conclude that, because of the enhanced diffractive cross sections for all $\left|\beta_{2}\right|>0$, all of the deformed core calculations move the theoretical value in the direction of the measured value, 82(16) $\mathrm{mb}$. More information is needed to inform the correct value of the mass $\beta_{2}$ to be used in the present strong interaction, inelastic excitations, context.

\section{CONCLUSIONS}

We have investigated the effects of including the dynamical reorientation and inelastic excitation of an assumed quadrupole deformed projectile core in the reaction mechanism of the single nucleon knockout reaction. Both the elastic breakup and stripping components of the inclusive knockout cross sections are treated nonperturbatively, within the eikonal approximation.

In the two model examples considered we find an enhancement of the diffractive breakup component of the cross section indicating that the core is dynamically active in the transfer of energy from the center of mass motion to excitation of the projectile. In contrast, the cross section for the essentially geometrical, absorptive stripping mechanism is found to be unaffected. Although in the present inclusive calculation we cannot apportion the additional diffractive cross section to the population of any specific final states, the magnitudes of the core dynamical effects are of the same order as the partial cross section that is observed to be missing in comparisons between spherical core calculations and selected, more exclusive, data sets.

We suggest therefore that the extension of the present work, to allow partial cross section calculations, would be very worthwhile and it is under consideration. Because we have clarified here that the dominant dynamical effects are on the elastic breakup cross section, then fully coupled channels, time-dependent, and other all-order breakup methodologies could also be used for this purpose.

\section{ACKNOWLEDGMENTS}

This work was supported by the United Kingdom Engineering and Physical Sciences Research Council (EPSRC) through Grant GR/M82141.
[1] P. G. Hansen and J. A. Tostevin, Annu. Rev. Nucl. Part. Sci. 53, 219 (2003).

[2] P. G. Hansen and B. M. Sherrill, Nucl. Phys. A693, 133 (2001).

[3] M. S. Hussein and K. W. McVoy, Nucl. Phys. A445, 124 (1985).

[4] J. A. Tostevin, Nucl. Phys. A682, 320c (2001).

[5] J. S. Al-Khalili, Nucl. Phys. A689, 551c (2001).

[6] T. Aumann et al., Phys. Rev. Lett. 84, 35 (2000).

[7] A. Sakharuk and V. Zelevinsky, Phys. Rev. C 61, 014609 (1999).

[8] F. M. Nunes, I. J. Thompson, and R. C. Johnson, Nucl. Phys. A596, 171 (1996).

[9] R. J. Glauber, in Lectures in Theoretical Physics, edited by W. E. Brittin (Interscience, New York, 1959), Vol. 1, p. 315.

[10] G. Fäldt and R. Glauber, Phys. Rev. C 42, 395 (1990).

[11] J. A. Christley and J. A. Tostevin, Phys. Rev. C 59, 2309 (1999).
[12] D. A. Varshalovich etal., Quantum Theory of Angular Momentum (World Scientific, Singapore, 1989).

[13] L. Ray, Phys. Rev. C 20, 1857 (1979).

[14] V. Maddalena et al., Phys. Rev. C 63, 024613 (2001).

[15] S. Raman, At. Data Nucl. Data Tables 36, 1 (1987).

[16] J. S. Al-Khalili, J. A. Tostevin, and I. J. Thompson, Phys. Rev. C 54, 1843 (1996).

[17] A. Ozawa et al., Nucl. Phys. A693, 32 (2001).

[18] S. K. Charagi and S. K. Gupta, Phys. Rev. C 41, 1610 (1990).

[19] N. Imai et al., Phys. Rev. Lett. 92, 062501 (2004).

[20] B. G. Harvey et al., Phys. Rev. 146, 712 (1966).

[21] S. Kubono, M. H. Tanaka, M. Sugitani, K. Morita, H. Utsunomiya, M. K. Tanaka, S. Shimoura, E. Takada, M. Fukada, and K. Takimoto, Phys. Rev. C 31, 2082 (1985). 